\title{
Functional wiring of hypocretin and LC-NE neurons: implications for arousal
}

\author{
Matthew E. Carter ${ }^{1 *}$, Luis de Lecea $^{2 *}$ and Antoine Adamantidis ${ }^{3 *}$ \\ ${ }^{1}$ Department of Biochemistry, University of Washington, Seattle, WA, USA \\ ${ }^{2}$ Department of Psychiatry and Behavioral Sciences, Stanford University, Stanford, CA, USA \\ ${ }^{3}$ Department of Psychiatry, Douglas Mental Health University Institute, McGill University, Montreal, QC, Canada
}

Edited by:

Benjamin Boutrel, Lausanne

University Hospital, Switzerland

Reviewed by:

Akihiro Yamanaka, Nagoya

University, Japan

Anne Vassalli, The Massachusetts

Institute of Technology, USA

\section{*Correspondence:}

Matthew E. Carter, Department of

Biochemistry, University of

Washington, Health Sciences

Building, Rm J661, Seattle,

WA 98103, USA.

e-mail: carterme@uw.edu;

Luis de Lecea, Department of

Psychiatry and Behavioral Sciences,

Stanford University, 1201 Welch

Road, MSLS Building, Rm 154,

Stanford, CA 94305, USA.

e-mail: llecea@stanford.edu;

Antoine Adamantidis, Department

of Psychiatry, Douglas Mental

Health University Institute, McGill

University, 6875 LaSalle Blvd,

Montréal, QC H4H 1R3, Canada.

e-mail: antoine.adamantidis@

mcgill.ca

Sleep and wakefulness are two mutually exclusive states that cycle with both ultradian and circadian periods throughout the animal kingdom. Wakefulness is a conscious state in which an animal can perceive and interact with its environment. After prolonged period of wakefulness, sleep pressure increases and leads to the onset of sleep that is characterized as a period of relative inactivity with stereotyped posture and higher sensory threshold.

In mammals, sleep is generally divided into slow-wave sleep (SWS, or NREM sleep in humans), and rapid eye movement (REM) sleep (also called "paradoxical sleep"). Wakefulness, SWS and REM sleep are distinct behavioral states that can be defined by precise electroencephalographic (EEG) and electromyographic (EMG) features. During wake, low-amplitude, mixed-frequency oscillations predominate. SWS is characterized by high-amplitude slow oscillations $(0.5-4 \mathrm{~Hz})$ whose predominance (as measured by the EEG power density) reflects the depth of sleep. REM sleep is a singular behavioral state, characterized by faster, mixed frequencies oscillations, among which theta $(5-10 \mathrm{~Hz})$ oscillations dominate in rodents, accompanied by muscle atonia, as well as fluctuation of the heart and breathing rates.

Although states of sleep and wakefulness are qualitatively and quantitatively easy to characterize, it is surprisingly difficult to define what is meant by "arousal." The term arousal usually refers to the degree of vigilance and alertness during wakefulness, manifesting as increased motor activation, responsiveness to sensory inputs, emotional reactivity, and enhanced cognitive processing.

The brain mechanisms underlying the organization of the sleep-wake cycle and general level of arousal remain unclear and many classical studies have identified several populations of neurons whose activity correlates with distinct behavioral states. It was originally assumed that neurons that are active before behavioral transitions (i.e., neurons active preceding a sleep-to-wake transition) promote the coming state, while neurons that are active during a specific state (wakefulness or sleep) are important to maintain it. This view is made more complicated by the understanding that neurons in a network may show state-boundaryassociated activity because of connectivity to other, more causal neurons without being directly responsible themselves for state transitions. Nevertheless, it has generally been inferred that there are neural populations that play a causal role in sleep and/or arousal states. Populations that are thought to promote arousal include: the hypocretin (hcrt-also called "orexins")expressing neurons in the lateral hypothalamus, the noradrenergic locus coeruleus (LC)-expressing neurons in the brainstem, 
the serotoninergic dorsal raphe nuclei (DRN) in the brainstem, the histaminergic tuberomammilary nucleus (TMN) in the posterior hypothalamus, the cholinergic pedunculopontine (PPT) and laterodorsal tegmental (LDT) nuclei in the midbrain, as well as cholinergic neurons in the basal forebrain (Jones, 2003). In contrast, inhibitory neurons from anterior hypothalamic structures are active during SWS, while Melanin-Concentrating Hormone $(\mathrm{MCH})$ neurons from the lateral hypothalamus, as well as glutamatergic and GABAergic neurons from the brainstem are active during REM sleep (Fort et al., 2009).

In recent years, we and others have begun using optogenetic technology with various mouse models to address questions such as How do arousal systems regulate wakefulness and arousal? How do they functionally interact to promote, maintain or broaden arousal in specific contexts? In our recent studies, we have been particularly interested in neurons that express hort (de Lecea et al., 1998; Sakurai et al., 1998). The hort are two neuro-excitatory peptides (de Lecea et al., 1998; Sakurai et al., 1998) produced in $\sim 3200$ neurons in the mouse lateral hypothalamus $(\sim 6700$ and 50,000-80,000 in the rat and human brain, respectively) (de Lecea and Sutcliffe, 2005; Modirrousta et al., 2005). These neurons receive functional inputs from multiple systems distributed in the cortex, limbic system, sub-cortical areas including the hypothalamus itself, thalamus, and ascending projections from the brainstem cholinergic nuclei, the reticular formation, the midbrain raphe nuclei, and the periaqueductal gray. In turn, these neurons project throughout the central nervous system, including to arousal and reward centers of the brain, to neurons expressing hcrt receptors (OX1R and OX2R). The afferent and efferent projections of hort neurons suggest that they play a role in multiple hypothalamic functions including regulating the sleep/wake cycle and goal-oriented behaviors. Interestingly, we have found that a specific efferent projection from hcrt neurons to noradrenergic LC neurons mediate sleep-to-wake transitions and possibly more general aspects of arousal.

Here, we summarize recent optogenetic experiments that test the hypothesis that hort and LC neurons cause arousal state transitions and maintenance (Adamantidis et al., 2007; Carter et al., 2009, 2010, 2012). First, we briefly highlight and summarize previous reports about these systems using traditional genetic and pharmacological techniques. Next, we integrate our own findings using optogenetic probes to selectively stimulate or inhibit these systems in freely-moving mice. Finally, we discuss unresolved questions and speculate on future anatomical and functional dissections of arousal circuits.

\section{HYPOCRETINS, WAKEFULNESS, AND NARCOLEPSY}

hcrt neurons are generally silent during quiet wakefulness, SWS, and REM sleep but show high discharge rates during active wake and REM sleep-to-wake transitions (Lee et al., 2005; Mileykovskiy et al., 2005; Takahashi et al., 2008; Hassani et al., 2009). In addition, they show high discharge rates during arousal elicited by environmental stimuli (e.g., an auditory stimulus) (Takahashi et al., 2008) and goal-oriented behavior (Mileykovskiy et al., 2005; Takahashi et al., 2008). These studies suggest that hort neurons participate to sleep-to-wake transitions, as well as in the increased alertness observed during various goal-oriented behaviors.
Blockade or suppression of hort signaling demonstrates the necessity of hort for the integrity of behavioral states in mice, rats, dogs, humans, and possibly zebrafishes (Sakurai, 2007; Yokogawa et al., 2007). Indeed, the most compelling loss-offunction evidence comes from the link between hcrt deficiency and the symptoms of narcolepsy (Peyron et al., 2000; Saper et al., 2010). Narcoleptic patients with cataplexy have a complete absence of hcrt gene transcripts in the hypothalamus as well as non- or barely-detectable levels of hort in the cerebrospinal fluid (Thannickal et al., 2000; Sakurai, 2007; Yokogawa et al., 2007). Doberman narcoleptic dogs bear a mutation in $O X 2 R$, and all genetically engineered rodents with either a deletion of hcrt, OX2R, or hcrt cells present behavioral arrests that resemble cataplexy, the hallmark of narcolepsy (Jones, 2003; Sakurai, 2007; Sehgal and Mignot, 2011). Importantly, genetic rescue of hcrt gene expression alleviated narcolepsy symptoms in mice (Liu et al., 2011; Blanco-Centurion et al., 2013).

Intracerebroventricular (i.c.v.) infusion of hcrt peptides or hcrt agonists causes an increase in the time spent awake and a decrease in SWS and REM sleep [review in Sakurai (2007)]. Stereotactic injection of the peptide in the LC, LDT, basal forebrain, or the lateral hypothalamus increased wakefulness and locomotor activity often associated with a marked reduction in SWS and REM sleep (Hagan et al., 1999). More recently, genetic dis-inhibition of hort neurons using a selective GABA-B receptor gene deletion only in hort neurons induced severe fragmentation of sleep/wake states during both the light and dark periods without showing an abnormality in total sleep/wake durations or signs of cataplexy (Matsuki et al., 2009). Collectively, these data suggest that the hort peptides are important to define boundaries between sleep and wake states, as shown by the fragmentation of sleep and wake state in animal models of narcolepsy.

Although it is widely documented that the biological function of hcrt peptides is necessary to maintain appropriate arousal and sleep, it remains unclear which of the two hcrt receptors, OX1R, or OX2R, is biologically responsible for hcrt's effects on arousal, as well as sleep stability and muscle tone control. OX1R mRNA is expressed in many brain areas, in particular the LC, raphe nuclei, LDT while $O X 2 R$ mRNA shows a complementary pattern of expression in cerebral cortex, raphe nuclei, as well as dorsomedial and posterior (in the tuberomammillary nucleus) hypothalamus (Trivedi et al., 1998; Marcus et al., 2001; Mieda et al., 2011). Thus, it has been proposed that the control of wakefulness and NREM sleep-to-wake depends critically on OX2R (Mochizuki et al., 2011) while the dysregulation of REM sleep (unique to narcolepsy-cataplexy) results from loss of signaling through both OX1R and OX2R (Mieda et al., 2011). However, their implications in the regulation of narcolepsy, in particular cataplexy and sleep attack, remain unclear. Dogs with heritable narcolepsy bear a null mutation in the OX2R gene (Lin et al., 1999) and the corresponding mouse model, the $O X 2 R \mathrm{KO}$ mice, show less severe symptoms than the dogs (Willie et al., 2003). Although OX1R participates to the regulation of arousal (Mieda et al., 2011), its contribution to the symptoms of narcolepsy remains to be further characterized.

Importantly, activity in other arousal systems is strongly perturbed during cataplexy. LC neurons cease discharge (Gulyani 
et al., 1999) and serotoninergic neurons significantly decrease their activity ( $\mathrm{Wu}, 2004)$, while cells located in the amygdala (Gulyani et al., 2002) and the TMN showed an increased level of firing (John et al., 2004). This association suggests that both OX1R (LC, raphe), and OX2R (TMN, raphe) are involved in the maintenance of appropriate muscle tone. Recent studies also highlighted the role of altered cholinergic systems in triggering cataplexy in narcoleptic mice (Kalogiannis et al., 2011, 2010). Therefore, an important, unresolved goal is to identify the functional wiring of hort neurons, as well as the dynamics of synaptic release from hcrt terminals to precisely delineate the downstream projections (de Lecea et al., 2012) that control arousal, sleep states, muscle tone, and goal-oriented behaviors.

\section{THE LOCUS COERULEUS, NOREPINEPHRINE, AND AROUSAL}

The LC is adjacent to the $4^{\text {th }}$ ventricle in the brainstem and contains neurons that synthetize the monoamine norepinephrine (NE). Although four other cell populations also produce NE (the A1, A2, A5, and A7 cell groups), the LC produces $~ 50 \%$ of the brain's total $\mathrm{NE}$ and is the only source to the cortex. There are many functional NE receptors located throughout the brain, with $\alpha 1$ and $\beta$ receptors usually causing excitatory postsynaptic potentials and $\alpha 2$ receptors usually causing inhibitory postsynaptic potentials. $\alpha 2$ receptors are densely found on LC neurons (Berridge and Waterhouse, 2003) themselves and serve as inhibitory autoreceptors to suppress intrinsic activity.

Recordings in awake behaving animals show that LC neurons fire tonically at $1-3 \mathrm{~Hz}$ during awake states, fire less during SWS sleep, and are virtually silent during REM sleep (Aston-Jones and Bloom, 1981; Jones, 2003; Saper et al., 2010). The LC also fires phasically in short bursts of $8-10 \mathrm{~Hz}$ during the presentation of salient stimuli that may increase wake duration. Like hcrt neurons, alterations in discharge rate precede changes in sleepto-wake transitions (Aston-Jones and Bloom, 1981), suggesting that these cells are important for transitions to wakefulness or attention.

Interestingly, physical lesions of the LC do not elicit consistent changes in cortical EEG or behavioral indices of arousal (Lidbrink, 1974; Blanco-Centurion et al., 2007). Genetic ablation of dopamine beta-hydroxylase, an enzyme required for NE synthesis, also does not disrupt sleep-wake states (Hunsley et al., 2006). This suggests the presence of redundant neural circuitry, external to the LC structure, supporting cortical activity and compensatory developmental mechanisms, respectively. However, central injections of pharmacological antagonists of $\alpha 1$ and $\beta$ noradrenergic receptors (Berridge and España, 2000) or agonists of inhibitory $\alpha 2$ autoreceptors (De Sarro et al., 1987) have substantial sedative effects. Central administration of NE directly into the ventricles or forebrain promotes wakefulness (Segal and Mandell, 1970; Flicker and Geyer, 1982). Stimulation of neurons in the LC using local microinjections of a cholinergic agonist (bethanechol) produces rapid activation of the forebrain EEG in halothane-anesthetized rats (Berridge and Foote, 1991). Recently, the LC-NE system was shown to be critical for maintaining the increased membrane potential of cortical neurons in awake compared to sleep states (Constantinople and Bruno,
2011). Taken together, these studies imply that the LC-NE system desynchronizes cortical activity and increases cortical membrane potential to increase arousal.

\section{OPTOGENETIC DISSECTION OF hcrt AND LC-NE CONTROL OF AROUSAL}

The activity of hcrt and LC-NE neurons correlates with sleepto-wake transitions, however, it has been difficult to selectively stimulate or inhibit specific hcrt and LC-NE populations with a temporal resolution relevant to sleep or wakefulness episodes, and to achieve spatial selectivity to probe those cells without affecting surrounding cells or fibers-of-passage. In an effort to better understand the temporal dynamics of neural circuits of wakefulness, we recently applied optogenetics to reversibly and selectively manipulate the activity of hcrt and LC neurons in freely-moving animals (Adamantidis et al., 2007; Carter et al., 2009, 2010, 2012). Optogenetics uses actuator opsin molecules (e.g., channelrhodopsin-2 (ChR2) or halorhodopsin-NpHR) to selectively activate or silence genetically-targeted cells, respectively, with flashes of light at specific wavelength (Boyden et al., 2005). Further information about optogenetic technology can be found in many other excellent reviews (Zhang et al., 2006; Miesenbock, 2009; Scanziani and Häusser, 2009; Yizhar et al., 2011; Deisseroth, 2012).

To deliver these actuators to hcrt or LC neurons, we used lentiviral and cre-dependent adeno-associated viral (AAV) gene delivery tools, respectively, under the control of cell-type specific promoters (Adamantidis et al., 2007). To deliver light to the hcrt or LC field, we designed optical-neural interfaces in which optical fibers were chronically implanted on the mouse skull, as described elsewhere (Adamantidis et al., 2005, 2007; Aravanis et al., 2007; Zhang et al., 2010). Using this strategy, we were able to control hort neural activity both in vitro and in vivo with millisecond-precise optical stimulation (Adamantidis et al., 2007). The high temporal and spatial precision of stimulation allowed us to mimic the physiological range of hypocretin neuron discharge rates $(1-30 \mathrm{~Hz})$ (Hassani et al., 2009). Indeed, we used light pulse trains for our optogenetic stimulation that were based on parameters on the actual frequency analysis of hcrt neurons in vivo (this is also true for optogenetic control of LC-NE neurons described below). We found that direct unilateral optical stimulation of hcrt neurons increased the probability of transitions to wakefulness from either SWS or REM sleep (Figure 1A). Interestingly, high frequency optical stimulation $(5-30 \mathrm{~Hz}$ light pulse trains) reduced the latency to wakefulness whereas $1 \mathrm{~Hz}$ trains did not, suggesting a frequency-dependent synaptic release of neurotransmitter (glutamate) and neuromodulators, including hcrt or dynorphin from the terminals. We further showed that the effects of stimulating hort neurons could be blocked by injection of a OX1R antagonist or by genetic deletion of the hort gene, suggesting that hort peptides mediate, at least in part, optogenetically-induced sleep-to-wake transitions. These results show that hort release from hcrt-expressing neurons is necessary for the wake-promoting properties of these neurons. Importantly, these results demonstrate a causal link between hcrt neuron activation and sleep-to-wake transitions, consistent with previous correlative studies. This was further supported by the fact that 

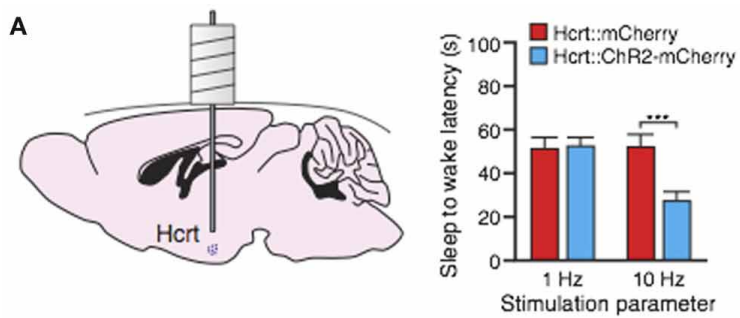

C

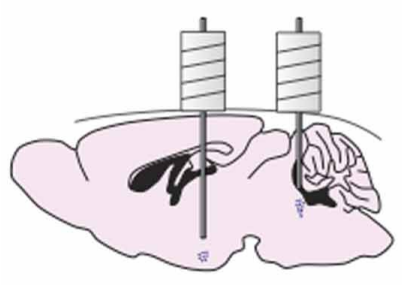

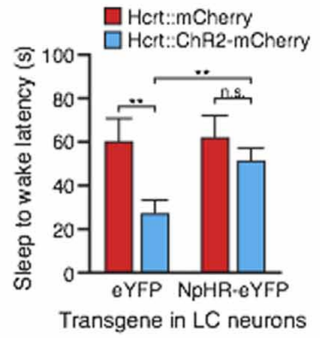

FIGURE 1|Optogenetic dissection of arousal circuits of the brain. (A) Stimulation of hort neurons with ChR2 causes a decrease in sleep-to-wake latency at $10 \mathrm{~Hz}$ but not $1 \mathrm{~Hz}$ (data from Adamantidis et al., 2007). (B) Stimulation of LC neurons with ChR2 causes immediate sleep-to-wake transitions at $10 \mathrm{~Hz}$ (data from Carter et al., 2010). (C) Stimulation of hert neurons at $10 \mathrm{~Hz}$ fails to
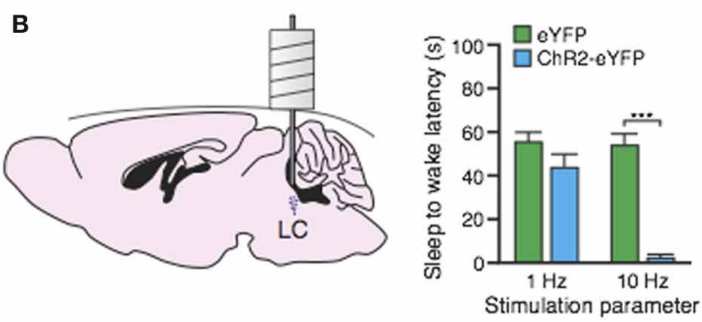

D
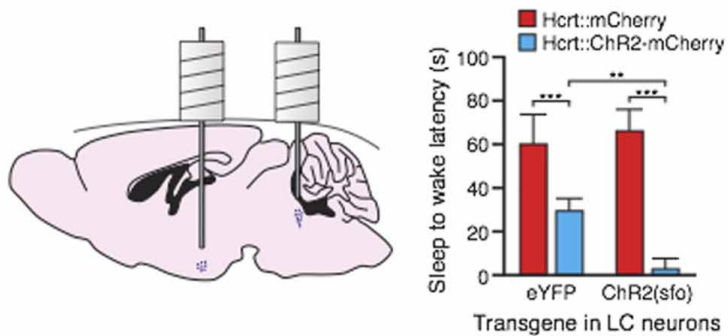

decrease sleep-to-wake latencies when the LC is concomitantly inhibited with NpHR (data from Carter et al., 2012). (D) Stimulation of the LC with a mutated version of ChR2 called a step-function-opsin (sfo) that increases membrane excitability enhances hcrt-mediated sleep-to-wake transitions (data from Carter et al., 2012). ${ }^{* *} P<0.01$; ${ }^{* * *} P<0.0001$. optical silencing of hcrt neurons promote SWS (Tsunematsu et al., 2011).

These results were recently confirmed by Sasaki and collaborators (Sasaki et al., 2011), who used a pharmacogenetic approach called Designer Receptors Exclusively Activated by Designer Drugs (DREADDs) to activate and suppress hort neural activity. DREADD technology allows bimodal modulation of neural activity with temporal resolution of several hours (Dong et al., 2010). They found that activation of hort neural activity increased wakefulness while suppression of hcrt activity promoted SWS.

In a second study (Carter et al., 2009), we demonstrated that hcrt control of sleep-wake transitions is under the dependence of sleep homeostasis processes since hcrt-mediated sleep-to-wake transitions are blocked by increased sleep pressure (caused by sleep deprivation). However, the effect of optogenetic stimulations of hcrt persisted in histamine decarboxylase knockout mice (mice that are unable to synthesize histamine) suggesting that another target that the histaminergic system is responsible for the effect of the hcrt. Finally, we showed that downstream arousal centers such as the LC neurons both increased their activity (as measured by c-Fos expression) in response to hcrt optogenetic stimulation. Because previous work showed an excitatory effect of hcrt on LC NE neurons (Bourgin et al., 2000), we investigated the hcrt-LC connection and focused our experimental investigations on the noradrenergic LC as a new target for optogenetic manipulation.

In a third study (Carter et al., 2010), we genetically targeted LC-NE neurons by stereotaxic injection of a Cre recombinasedependent adeno-associated virus (rAAV) into knock-in mice selectively expressing Cre in tyrosine hydroxylase $(\mathrm{TH})$ neurons
(Atasoy et al., 2008; Tsai et al., 2009). We found that both NpHR and ChR2 were functional and could inhibit and activate, respectively, LC-NE neurons both in vitro and in vivo (Figure 1B). We found that optogenetic low frequency $(1-10 \mathrm{~Hz})$ stimulation of LC-NE neurons caused immediate (less than $5 \mathrm{~s}$ ) sleep-towake transitions from both SWS and REM sleep. Stimulation of LC neurons during wakefulness increased locomotor activity and the total time spent awake, confirming the strong arousal effect. In contrast, NpHR-mediated silencing of LC-NE neurons decreased the duration of wake episodes but did not block sleepto-wake transitions when animals were asleep. Taken together, this study showed that activation of LC-NE neurons is necessary for maintaining normal durations of wakefulness (NpHR experiment), and sufficient to induce immediate sleep-to-wake transitions, sustained wakefulness, and increased locomotor arousal. Thus, we proposed that the LC-NE neurons act as a fast tuning system to promote sleep-to-wake transitions and general arousal. Interestingly, we found that sustained optical activation of LC-NE neurons induces locomotor arrest (Carter et al., 2010). Such behavioral arrests share common symptoms with cataplexy, catatonia or behavioral freezing both in animal models and human patients (Scammell et al., 2009). Possible mechanisms may involve NE depletion from LC-NE synapse terminals or LC-NE overexcitation of brainstem motor nuclei that would lead to paralysis. Further study is required to unravel the underlying mechanisms.

In our most recent study (Carter et al., 2012), we tested the hypothesis that LC activity gates hcrt neuron's effects on sleepto-wake transitions. Because hcrt and LC neural populations are located in distinct brain regions, it is physically possible to access both structures simultaneously in the same animal. We therefore 
took a dual optogenetic approach to stimulate hcrt neurons while concomitantly inhibiting or stimulating noradrenergic LC neurons during SWS sleep. We found that silencing LC neurons during hcrt stimulation blocked hcrt-mediated sleep-to-wake transitions (Figure 1C). In contrast, we found that increasing the excitability of LC neurons through step-function opsin (SFO) activation-which increase of target cells (Berndt et al., 2009) during hort stimulation (using a LC stimulation protocol that by itself does not increase sleep-to-wake transitions) enhanced hcrtmediated sleep-to-wake transitions (Figure 1D). Taken together, our results show that the LC serves as a necessary and sufficient downstream effector for hcrt-mediated SWS-to-wake transitions during the inactive period.

\section{hcrt AND LC-NE SYSTEM DYNAMICS}

Across our experimental studies, we observed that optogenetic manipulation of hcrt and LC-NE neurons affect sleep-to wake transitions with dramatically different temporal dynamics (Adamantidis et al., 2007; Carter et al., 2009, 2010, 2012). Acute optical activation of hcrt neurons causes sleep-to-wake transitions over a time period of 10-30s, while stimulation of LC neurons causes sleep-to-wake transitions in less than $5 \mathrm{~s}$. One explanation is that hort neurons may act as an upstream integrator of arousal during hypothalamic-related functions while the LC-NE system acts as a primary effector for arousal, stress, and attention. However, the neuronal effector systems are likely redundant and activated by distinct sets of inputs. Therefore, we cannot rule out that blocking other arousal systems, such as the central histaminergic and cholinergic systems, would also severely affect hcrt-induced behavioral state transitions in other experimental conditions.

Besides these short-term effects, it is also interesting that sustained (i.e., semi-chronic) photostimulation experiments of $\sim 1-4 \mathrm{~h}$ of hcrt neurons increased sleep-to-wake transitions without changing the total duration of wakefulness, whereas long-term photostimulation of LC-NE neurons significantly increased wakefulness duration. These results suggest that the hort system may regulate sleep-wake boundaries while LC-NE neurons may rather control wake duration by increasing cortical membrane potential and desynchronizing the cortical EEG.

The hypothalamic localization of hort neurons implies that these cells have a prominent arousal role during homeostatic processes, including sexual behavior, food foraging, stress response, and motivation. Besides their control of wakefulness, arousal

\section{REFERENCES}

Adamantidis, A., Thomas, E., Foidart, A., Tyhon, A., Coumans, B., Minet, A., et al. (2005). Disrupting the melanin-concentrating hormone receptor 1 in mice leads to cognitive deficits and alterations of NMDA receptor function. Eur. J. Neurosci. 21, 2837-2844.

Adamantidis, A. R., Zhang, F., Aravanis, A. M., Deisseroth, K., and de Lecea, L. (2007). Neural substrates of awakening probed with optogenetic

systems also participate in reward-seeking behavior, sexual activity, flight-or-fight responses, etc. This redundancy may have consolidated arousal function across evolution and diversified brain mechanisms supporting wakefulness and arousal-related behaviors necessary for survival. For example, activation of the LC-NE system increases arousal and cause anxiety-like behaviors (Itoi and Sugimoto, 2010). In contrast, the neuropeptide S (NPS) system, a peptide produced by a restricted neuronal population ventral to the LC, also increases arousal but decreases anxiety (Pape et al., 2010). Thus, to support such diverse behavioral functions, arousal circuits must have reached a high level of specification, possibly through a selective compartmentalization of their afferent and efferent connections, transmitters/modulators release capabilities and coherent activity with others arousal circuits.

\section{PERSPECTIVES}

In the past five years, the combination of optogenetics, genetically-engineered mouse models, and EEG/EMG analysis of sleep has provided a unique and powerful set of tools to further understand the contributions of the hort and LC systems to arousal, as well as other populations of neurons that regulate degrees of sleep and wakefulness. Targeting optogenetic probes to other populations of neurons in the brain will determine their individual and combined roles in sleep/wake boundaries. Furthermore, these tools will allow us to determine the brain mechanism underlying wake states based on anatomical projections, synaptic neurotransmission, and dynamics of transmitter release. The ability to target and selectively manipulate these circuits with high temporal precision $(<1 \mathrm{~s})$ further allows the possibility to investigate their role in a wide spectrum of behaviors such as food intake, addiction, stress, attention, and sexual arousal. Ultimately, these studies may unravel the pathophysiological mechanisms of psychiatric disorders such as chronic anxiety, addiction, attention deficit, and depression.

\section{ACKNOWLEDGMENTS}

Matthew E. Carter is supported by a fellowship from the Hilda and Preston Davis Foundation. Luis de Lecea is supported by grants from the Defense Advanced Research Projects Agency, the National Alliance for Research on Schizophrenia and Depression, and the Klarman Family Foundation. Antoine Adamantidis is supported by the Douglas Foundation, the Canadian Institute for Health Research, the Canadian Fund for Innovation, the Canadian Research Chair and the NSERC.

in behaving rats anticipates fluctuations in the sleep-waking cycle. J. Neurosci. 1, 876-886.

Atasoy, D., Aponte, Y., Su, H. H., and Sternson, S. M. (2008). A FLEX switch targets channelrhodopsin2 to multiple cell types for imaging and long-range circuit mapping. J. Neurosci. 28, 7025-7030.

Berndt, A., Yizhar, O., Gunaydin, L. A., Hegemann, P., and Deisseroth, K. (2009). Bi-stable neural state switches. Nat. Neurosci. 12, 229-234.

Berridge, C. W., and España, R. A. (2000). Synergistic sedative effects of noradrenergic alpha(1)- and beta-receptor blockade on forebrain electroencephalographic and behavioral indices. Neuroscience 99, 495-505.

Berridge, C. W., and Foote, S. L. (1991). Effects of locus coeruleus activation on electroencephalographic activity in neocortex and 
hippocampus. J. Neurosci. 11, 3135-3145.

Berridge, C. W., and Waterhouse, B. D. (2003). T he locus coeruleusnoradrenergic system: modulation of behavioral state and statedependent cognitive processes. Brain Res. Rev. 42, 33-84.

Blanco-Centurion, C., Gerashchenko, D., and Shiromani, P. J. (2007). Effects of saporin-induced lesions of three arousal populations on daily levels of sleep and wake. J. Neurosci. 27, 14041-14048.

Blanco-Centurion, C., Liu, M., Konadhode, R., Pelluru, D., and Shiromani, P. J. (2013). Effects of orexin gene transfer in the dorsolateral pons in orexin knockout mice. Sleep 36, 31-40.

Bourgin, P., Huitrón-Résendiz, S., Spier, A. D., Fabre, V., Morte, B., Criado, J. R., et al. (2000). Hypocretin-1 modulates rapid eye movement sleep through activation of locus coeruleus neurons. J. Neurosci. 20, 7760-7765.

Boyden, E. S., Zhang, F., Bamberg, E., Nagel, G., and Deisseroth, K. (2005). Millisecond-timescale, genetically targeted optical control of neural activity. Nat. Neurosci. 8 , 1263-1268.

Carter, M. E., Adamantidis, A., Ohtsu, H., Deisseroth, K., and de Lecea, L. (2009). Sleep homeostasis modulates hypocretin-mediated sleep-towake transitions. J. Neurosci. 29, 10939-10949.

Carter, M. E., Brill, J., Bonnavion, P., Huguenard, J. R., Huerta, R., and de Lecea, L. (2012). Mechanism for Hypocretin-mediated sleep-towake transitions. Proc. Natl. Acad. Sci. U.S.A. 109, E2635-E2644.

Carter, M. E., Yizhar, O., Chikahisa, S., Nguyen, H., Adamantidis, A., Nishino, S., et al. (2010). Tuning arousal with optogenetic modulation of locus coeruleus neurons. Nat. Publishing Group 13, 1526-1533.

Constantinople, C. M., and Bruno, R. M. (2011). Effects and mechanisms of wakefulness on local cortical networks. Neuron 69, 1061-1068.

Deisseroth, K. (2012). Optogenetics and psychiatry: applications, challenges, and opportunities. BPS 71, 1030-1032.

de Lecea, L., Carter, M. E., and Adamantidis, A. (2012). Shining light on wakefulness and arousal. BPS 71, 1046-1052.

de Lecea, L., Kilduff, T. S., Peyron, C., Gao, X., Foye, P. E., Danielson, P. E., et al. (1998). The hypocretins: hypothalamus-specific peptides with neuroexcitatory activity. Proc. Natl. Acad. Sci. U.S.A. 95, 322-327.

de Lecea, L., and Sutcliffe, J. G. (2005). Hypocretins. New York, NY: Springer Verlag.

De Sarro, G. B., Ascioti, C., Froio, F., Libri, V., and Nisticò, G. (1987). Evidence that locus coeruleus is the site where clonidine and drugs acting at alpha 1- and alpha 2-adrenoceptors affect sleep and arousal mechanisms. $\mathrm{Br}$. J. Pharmacol. 90, 675-685.

Dong, S., Rogan, S. C., and Roth, B. L. (2010). Directed molecular evolution of DREADDs: a generic approach to creating nextgeneration RASSLs. Nat. Protoc. 5, 561-573.

Flicker, C., and Geyer, M. A. (1982). The hippocampus as a possible site of action for increased locomotion during intracerebral infusions of norepinephrine. Behav. Neural Biol. 34, 421-426.

Fort, P., Bassetti, C. L., and Luppi, P.-H. (2009). Alternating vigilance states: new insights regarding neuronal networks and mechanisms. Eur. J. Neurosci. 29, 1741-1753.

Gulyani, S., Wu, M. F., Nienhuis, R., John, J., and Siegel, J. M. (2002). Cataplexy-related neurons in the amygdala of the narcoleptic dog. Neuroscience 112, 355-365.

Gulyani, S. A., Yau, E., Mignot, E., Phan, B., and Siegel, J. M. (1999). Locus coeruleus neurons: cessation of activity during cataplexy. Neuroscience 91, 1389-1399.

Hagan, J. J., Leslie, R. A., Patel, S., Evans, M. L., Wattam, T. A., Holmes, S., et al. (1999). Orexin A activates locus coeruleus cell firing and increases arousal in the rat. Proc. Natl. Acad. Sci. U.S.A. 96, 10911-10916.

Hassani, O. K., Lee, M. G., and Jones, B. E. (2009). Melanin-concentrating hormone neurons discharge in a reciprocal manner to orexin neurons across the sleep-wake cycle. Proc. Natl. Acad. Sci. U.S.A. 106 2418-2422.

Hunsley, M. S., Curtis, W. R., and Palmiter, R. D. (2006). Behavioral and sleep/wake characteristics of mice lacking norepinephrine and hypocretin. Genes Brain Behav. 5, 451-457.

Itoi, K., and Sugimoto, N. (2010). The brainstem noradrenergic systems in stress, anxiety and depression. J. Neuroendocrinol. 22, 355-361.

John, J., Wu, M.-F., Boehmer, L. N., and Siegel, J. M. (2004). Cataplexyactive neurons in the hypothalamus. Neuron 42, 619-634.
Jones, B. E. (2003). Arousal systems. Front. Biosci. 8, s438-s451.

Kalogiannis, M., Grupke, S. L., Potter, P. E., Edwards, J. G., Chemelli, R. M., Kisanuki, Y. Y., et al. (2010). Narcoleptic orexin receptor knockout mice express enhanced cholinergic properties in laterodorsal tegmental neurons. Eur. J. Neurosci. 32, 130-142.

Kalogiannis, M., Hsu, E., Willie, J. T., Chemelli, R. M., Kisanuki, Y. Y., Yanagisawa, M., et al. (2011). Cholinergic modulation of narcoleptic attacks in double orexin receptor knockout mice. PLOS ONE 6:e18697. doi: 10.1371/journal.pone.0018697

Lee, M. G., Hassani, O. K., and Jones, B. E. (2005). Discharge of identified orexin/hypocretin neurons across the sleep-waking cycle. J. Neurosci. 25, 6716-6720.

Lidbrink, P. (1974). The effect of lesions of ascending noradrenaline pathways on sleep and waking in the rat. Brain Res. 74, 19-40.

Lin, L., Faraco, J., Li, R., Kadotani, H. Rogers, W., Lin, X., et al. (1999). The sleep disorder canine narcolepsy is caused by a mutation in the hypocretin (orexin) receptor 2 gene. Cell 98, 365-376.

Liu, M., Blanco-Centurion, C. Konadhode, R., Begum, S., Pelluru, D., Gerashchenko, D., et al. (2011). Orexin gene transfer into zona incerta neurons suppresses muscle paralysis in narcoleptic mice. J. Neurosci. 31, 6028-6040.

Marcus, J. N., Aschkenasi, C. J., Lee, C. E., Chemelli, R. M., Saper, C. B., Yanagisawa, M., et al. (2001) Differential expression of orexin receptors 1 and 2 in the rat brain. J. Comp. Neurol. 435, 6-25.

Matsuki, T., Nomiyama, M., Takahira, H., Hirashima, N., Kunita, S., Takahashi, S., et al. (2009). Selective loss of GABA(B) receptors in orexin-producing neurons results in disrupted sleep/wakefulness architecture. Proc. Natl. Acad. Sci. U.S.A. 106, 4459-4464.

Mieda, M., Hasegawa, E., Kisanuki, Y. Y., Sinton, C. M., Yanagisawa, M., and Sakurai, T. (2011). Differential roles of orexin receptor-1 and 2 in the regulation of non-REM and REM sleep. J. Neurosci. 31, 6518-6526.

Miesenbock, G. (2009). The optogenetic catechism. Science 326 , 395-399.

Mileykovskiy, B. Y., Kiyashchenko, L. I., and Siegel, J. M. (2005). Behavioral correlates of activity in identified hypocretin/orexin neurons. Neuron $46,787-798$.
Mochizuki, T., Arrigoni, E., Marcus, J. N., Clark, E. L., Yamamoto, M., Honer, M., et al. (2011). Orexin receptor 2 expression in the posterior hypothalamus rescues sleepiness in narcoleptic mice. Proc. Natl. Acad. Sci. U.S.A. 108, 4471-4476.

Modirrousta, M., Mainville, L., and Jones, B. E. (2005). Orexin and $\mathrm{MCH}$ neurons express c-Fos differently after sleep deprivation vs. recovery and bear different adrenergic receptors. Eur. J. Neurosci. 21, 2807-2816.

Pape, H.-C., Jüngling, K., Seidenbecher, T., Lesting, J., and Reinscheid, R. K. (2010). Neuropeptide S: a transmitter system in the brain regulating fear and anxiety. Neuropharmacology 58, 29-34.

Peyron, C., Faraco, J., Rogers, W., Ripley, B., Overeem, S., Charnay, Y., et al.. (2000). A mutation in a case of early onset narcolepsy and a generalized absence of hypocretin peptides in human narcoleptic brains. Nat. Med. 6, 991-997.

Sakurai, T. (2007). The neural circuit of orexin (hypocretin): maintaining sleep and wakefulness. Nat. Rev. Neurosci. 8, 171-181.

Sakurai, T., Amemiya, A., Ishii, M., Matsuzaki, I., Chemelli, R. M., Tanaka, H., et al. (1998). Orexins and orexin receptors: a family of hypothalamic neuropeptides and G protein-coupled receptors that regulate feeding behavior. Cell 92, 573-585.

Saper, C. B., Fuller, P. M., Pedersen, N. P., Lu, J., and Scammell, T. E. (2010). Sleep state switching. Neuron 68, 1023-1042.

Sasaki, K., Suzuki, M., Mieda, M., Tsujino, N., Roth, B., and Sakurai, T. (2011). Pharmacogenetic modulation of orexin neurons alters sleep/wakefulness states in mice. PLoS ONE 6:e20360. doi: 10.1371/journal.pone. 0020360

Scammell, T. E., Willie, J. T., Guilleminault, C., Siegel, J. M., and International Working Group on Rodent Models of Narcolepsy. (2009). A consensus definition of cataplexy in mouse models of narcolepsy. Sleep 32, 111-116.

Scanziani, M., and Häusser, M. (2009). Electrophysiology in the age of light. Nature 461, 930-939.

Segal, D. S., and Mandell, A. J. (1970). Behavioral activation of rats during intraventricular infusion of norepinephrine. Proc. Natl. Acad. Sci. U.S.A. 66, 289-293.

Sehgal, A., and Mignot, E. (2011). Genetics of sleep and sleep disorders. Cell 146, 194-207. 
Takahashi, K., Lin, J.-S., and Sakai, K. (2008). Neuronal activity of orexin and non-orexin waking-active neurons during wake-sleep states in the mouse. Neuroscience 153, 860-870.

Thannickal, T. C., Moore, R. Y., Nienhuis, R., Ramanathan, L., Gulyani, S., Aldrich, M., et al. (2000). Reduced number of hypocretin neurons in human narcolepsy. Neuron 27, 469-474.

Trivedi, P., Yu, H., MacNeil, D. J., Van der Ploeg, L. H., and Guan, X. M. (1998). Distribution of orexin receptor mRNA in the rat brain. FEBS Lett. 438, 71-75.

Tsai, H.-C., Zhang, F., Adamantidis, A., Stuber, G. D., Bonci, A., de Lecea, L., et al. (2009). Phasic firing in dopaminergic neurons is sufficient for behavioral conditioning. Science 324, 1080-1084.

Tsunematsu, T., Kilduff, T. S., Boyden, E. S., Takahashi, S.,
Tominaga, M., and Yamanaka, A. (2011). Acute optogenetic silencing of orexin/hypocretin neurons induces slow-wave sleep in mice. J. Neurosci. 31, 10529-10539.

Willie, J. T., Chemelli, R. M., Sinton, C. M., Tokita, S., Williams, S. C., Kisanuki, Y. Y., et al. (2003). Distinct narcolepsy syndromes in Orexin receptor-2 and Orexin null mice: molecular genetic dissection of Non-REM and REM sleep regulatory processes. Neuron 38, 715-730.

Wu, M. F. (2004). Activity of dorsal raphe cells across the sleep-waking cycle and during cataplexy in narcoleptic dogs. J. Physiol. (Lond.) 554, 202-215.

Yizhar, O., Fenno, L. E., Davidson, T. J., Mogri, M., and Deisseroth, K. (2011). Optogenetics in neural systems. Neuron 71, 9-34.
Yokogawa, T., Marin, W., Faraco, J., Pézeron, G., Appelbaum, L., Zhang, J., et al. (2007). Characterization of sleep in zebrafish and insomnia in hypocretin receptor mutants. PLoS Biol. 5:e277. doi: 10.1371/journal.pbio. 0050277

Zhang, F., Gradinaru, V., Adamantidis, A. R., Durand, R., Airan, R. D., de Lecea, L., et al. (2010). Optogenetic interrogation of neural circuits: technology for probing mammalian brain structures. Nat. Protoc. 5, 439-456.

Zhang, F., Wang, L.-P., Boyden, E. S., and Deisseroth, K. (2006). Channelrhodopsin-2 and optical control of excitable cells. Nat. Methods 3, 785-792.

Conflict of Interest Statement: The authors declare that the research was conducted in the absence of any commercial or financial relationships that could be construed as a potential conflict of interest.

Received: 12 January 2013; accepted: 23 April 2013; published online: 20 May 2013.

Citation: Carter ME, de Lecea L and Adamantidis A (2013) Functional wiring of hypocretin and LC-NE neurons: implications for arousal. Front. Behav. Neurosci. 7:43. doi: 10.3389/ fnbeh.2013.00043

Copyright (C) 2013 Carter, de Lecea and Adamantidis. This is an openaccess article distributed under the terms of the Creative Commons Attribution License, which permits use, distribution and reproduction in other forums, provided the original authors and source are credited and subject to any copyright notices concerning any third-party graphics etc. 